\title{
Accurate Low-Frequency Approximation for Wires within a Two-Layered Earth
}

\section{Blagoja Markovski, Leonid Grcev, Vesna Arnautovski-Toseva, Andrijana Kuhar}

Ss. Cyril and Methodius University in Skopje, Faculty of Electrical Engineering and Information Technologies, Rugjer Boshkovik 18, 1000 Skopje, Macedonia, bmarkovski@feit.ukim.edu.mk, lgrcev@feit.ukim.edu.mk, atvesna@feit.ukim.edu.mk, kuhar@feit.ukim.edu.mk

\begin{abstract}
Rigorous electromagnetic models and approximations are traditionally based on the Sommerfeld's resolution for Hertz vector potentials. However, another resolution, based on transverse Hertz vector potentials, also exists. This paper shows that a low frequency approximation, based on this resolution, for wires embedded in a two-layered earth, is more accurate than the existing alternative. The accuracy of proposed approximation is validated for a range of different wire geometries, frequencies and earth characteristics.
\end{abstract}

Keywords: Electromagnetic model; Grounding; Green's functions; Modeling

\section{Introduction}

Analysis of wires embedded in earth, for frequency ranges from DC to tens of $\mathrm{MHz}$, is of interest in a number of engineering analyses, such as those related to grounding in power systems [1], EMC [2], lightning protection [3] and subsurface communications [4]. The electromagnetic model based on the mixed potential integral equation (MPIE) [5] and the method of moments [6], is generally the preferred choice for such analyses.

When the corresponding Green's functions are based of mathematically exact solution for the electric field in planar-layered media, electromagnetic modeling involves evaluation of Sommerfeld-type integrals by complex numerical procedures. Alternatively, simpler models for analysis with reasonable loss of accuracy can be obtained by approximating the mathematically exact equations. Amongst existing variants, the low-frequency (LF) or often cited image approximations in analytical form, are mostly preferred in practice, due to their simplicity, ease in development and implementation. 
It is important to note that existing image approximations are based on the Sommerfeld's resolution for Hertz vector potentials from horizontal electric dipole [7], also known as traditional choice of potentials. This resolution is widely accepted in electromagnetic modeling of grounding systems, antenna theory and other EMC related studies. However, other resolutions also exist [8], for example the transverse resolution or so-called alternative choice of potentials. Both resolutions are basis for development of different formulations of potentials in MPIE [9]. Recent analysis for wires embedded in uniform earth have shown that LF approximation derived from the transverse resolution for potentials is substantially more accurate than other image approximations based on Sommerfeld's resolution [10].

In this paper, we propose new LF approximation of the Green's functions for MPIE modeling of wires within a single layer in two-layered earth. This approximation is derived from a rigorous full-wave solution based on the alternative choice of potentials for planar-layered media [11], implemented in formulation A of potentials in MPIE. The proposed approximation is compared with the existing image approximation for a two-layered earth, which is based on the traditional formulation of potentials, for a range of different wire geometries, frequencies and earth characteristics. The accuracy of the proposed and the existing approximations are evaluated by comparison with results obtained by the commercial electromagnetic simulation software FEKO. This software incorporates an exact Sommerfeld integral formulation for analysis of wires in a layered media [12].

\section{Formulation of Electric Field by MPIE}

In MPIE, the scattered electric field vector $\vec{E}^{s}(\vec{r})$ from a straight thin wire with longitudinal current $I\left(\vec{r}^{\prime}\right)$, can be expressed in terms of magnetic vector and electric scalar potentials, $\vec{A}(\vec{r})$ and $\Phi(\vec{r})$ respectively:

$$
\begin{aligned}
& \vec{E}_{m, i}^{S}(\vec{r})=-j \omega \vec{A}_{m, i}(\vec{r})-\nabla \Phi_{m, i}(\vec{r}) \\
& \vec{A}_{m, i}(\vec{r})=\frac{\mu_{m}}{4 \pi} \int_{\ell^{\prime}} \bar{G}_{A_{m, i}}\left(\vec{r}, \vec{r}^{\prime}\right) I\left(\vec{r}^{\prime}\right) d \ell^{\prime} \\
& \Phi_{m, i}(\vec{r})=\frac{j \omega}{4 \pi \underline{\sigma}_{i}} \int_{\ell^{\prime}} G_{\Phi_{m, i}}\left(\vec{r}, \vec{r}^{\prime}\right) q\left(\vec{r}^{\prime}\right) d \ell^{\prime} \\
& q\left(\vec{r}^{\prime}\right)=\frac{-1}{j \omega} \nabla^{\prime} I\left(\vec{r}^{\prime}\right)
\end{aligned}
$$


where $\overline{\bar{G}}_{A_{m, i}}$ and $G_{\Phi_{m, i}}$ are dyadic magnetic vector and electric scalar potential Green's functions for source in layer $m$ and observation point in layer $i$. The position of electric dipole with strength $I\left(\vec{r}^{\prime}\right) d \ell^{\prime}$, on the axis $\ell^{\prime}$ of a $\left(\hat{x}^{\prime}, \hat{y}^{\prime}, \hat{z}^{\prime}\right)$ directed straight wire is denoted by $\vec{r}^{\prime}$, and position of the observation point for $(\hat{x}, \hat{y}, \hat{z})$ - directed electric field vector is denoted by $\vec{r}$.

Green's functions for potentials $\overline{\bar{G}}_{A_{m, i}}$ and $G_{\phi_{m, i}}$ in MPIE are not unique. Among several possibilities, the Green's functions for the traditional formulation and formulation A of potentials, and their LF approximations for source and observation points within same layer are of particular interest for the analysis in this paper.

The traditional formulation is based on the Sommerfeld's resolution [7] that postulates that for source and observation points within same layer, $x$ - directed horizontal electric dipole (HED) has $x$ - and $z$ - component of magnetic vector potential, as illustrated on Fig. 1a). Formulation A is based on the transverse resolution [8], where $y$-component accompanies the primary $x$-component, as illustrated on Fig. 1b). The $z$ - components of magnetic vector potentials in both formulations are identical for source and observation points in same layer, and the scalar potential Green's function for formulation A is identical with the scalar potential Green's function for vertical electric dipole (VED) from the traditional formulation.

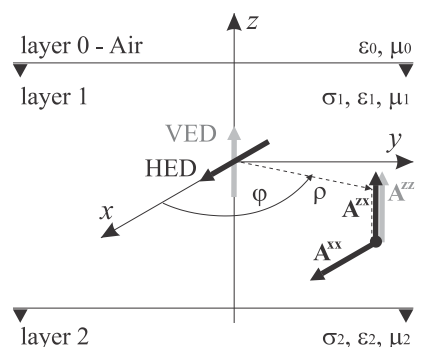

a)

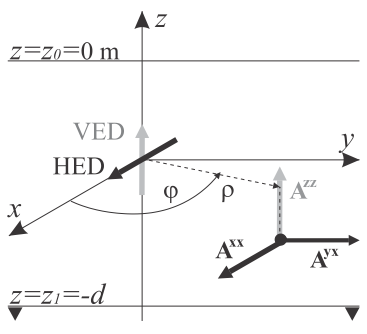

b)

Figure 1

Components of magnetic vector potentials associated to HED and VED for source and observation points within same layer in two-layered earth for: (a) traditional formulation and (b) formulation A

Green's functions for traditional formulation of potentials can be obtained from [13], while exact mathematical solution for spatial domain Green's functions for formulation $\mathrm{A}$ and related parameters for general planar-layered media are provided in Annex A. These Green's functions are basis for development of the corresponding LF approximations for a two-layered earth. 


\section{LF Approximations for Source and Observation Points within Same Layer in Two-layered Earth}

\subsection{Traditional Formulation of Potentials in MPIE}

Dyadic Green's function for magnetic vector potential is expressed as:

$$
\overline{\bar{G}}_{A}=\left(\hat{x}^{\prime} \hat{x}+\hat{y}^{\prime} \hat{y}\right) G_{A}^{x x}+\hat{x}^{\prime} \hat{z} G_{A}^{z x}+\hat{y}^{\prime} \bar{z} G_{A}^{z y}+\hat{z}^{\prime} \hat{z} G_{A}^{z z}
$$

Details of development of LF approximations for traditional formulation of potentials can be found in [14]-[15], and here they are rewritten for completeness:

$$
\begin{aligned}
& G_{A(L F)}^{x x}=\left\{\begin{array}{l}
g_{d}^{0} \\
g_{d}^{1} \\
g_{d}^{2}
\end{array}\right. \\
& \text {, } m=0 \\
& \text {, } m=1 \\
& \text {, } m=2
\end{aligned}
$$

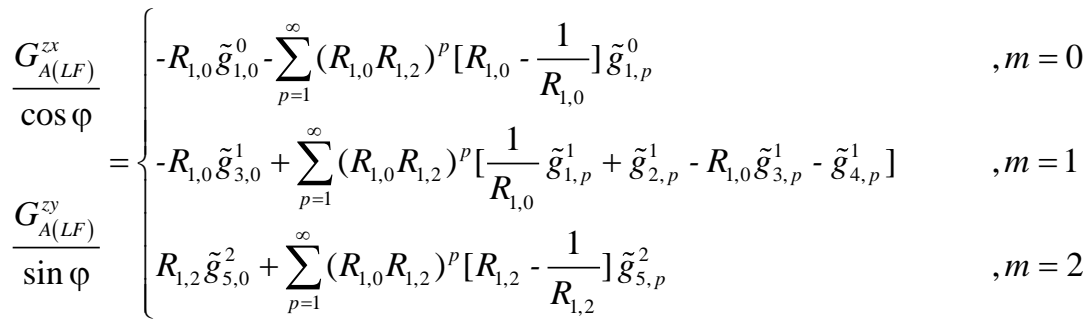

$$
\begin{aligned}
& G_{A(L F)}^{z z}= \begin{cases}g_{d}^{0}+R_{1,0} g_{1,0}^{0}+\sum_{p=1}^{\infty}\left(R_{1,0} R_{1,2}\right)^{p}\left[R_{1,0}-\frac{1}{R_{1,0}}\right] g_{1, p}^{0} & , m=0 \\
g_{d}^{1}-R_{1,0} g_{3,0}^{1}+\sum_{p=1}^{\infty}\left(R_{1,0} R_{1,2}\right)^{p}\left[-\frac{1}{R_{1,0}} g_{1, p}^{1}+g_{2, p}^{1}-R_{1,0} g_{3, p}^{1}+g_{4, p}^{1}\right] & , m=1 \\
g_{d}^{2}+R_{1,2} g_{5,0}^{2}+\sum_{p=1}^{\infty}\left(R_{1,0} R_{1,2}\right)^{p}\left[R_{1,2}-\frac{1}{R_{1,2}}\right] g_{5, p}^{2} & , m=2\end{cases} \\
& G_{\Phi(L F)}= \begin{cases}g_{d}^{0}-R_{1,0} g_{1,0}^{0}-\sum_{p=1}^{\infty}\left(R_{1,0} R_{1,2}\right)^{p}\left[R_{1,0}-\frac{1}{R_{1,0}}\right] g_{1, p}^{0} & , m=0 \\
g_{d}^{1}+R_{1,0} g_{3,0}^{1}+\sum_{p=1}^{\infty}\left(R_{1,0} R_{1,2}\right)^{p}\left[\frac{1}{R_{1,0}} g_{1, p}^{1}+g_{2, p}^{1}+R_{1,0} g_{3, p}^{1}+g_{4 p}^{1}\right] & , m=1 \\
g_{d}^{2}-R_{1,2} g_{5,0}^{2}-\sum_{p=1}^{\infty}\left(R_{1,0} R_{1,2}\right)^{p}\left[R_{1,2}-\frac{1}{R_{1,2}}\right] g_{5, p}^{2} & , m=2\end{cases}
\end{aligned}
$$

Details for $R_{m, m \pm 1}, g_{d}^{m}, g_{l, p}^{m}$ and $\tilde{g}_{l, p}^{m}$ are provided in Appendices A and B. 


\subsection{Formulation A of Potentials in MPIE}

Dyadic Green's function for magnetic vector potential is expressed as:

$$
\overline{\bar{K}}_{A}=\hat{x}^{\prime} \hat{x} K_{A}^{x x}+\hat{y}^{\prime} \hat{y} K_{A}^{y y}+\left(\hat{x}^{\prime} \hat{y}+\hat{y}^{\prime} \hat{x}\right) K_{A}^{y x}+\hat{z}^{\prime} \hat{x} K_{A}^{x z}+\hat{z}^{\prime} \hat{y} K_{A}^{y z}+\hat{z}^{\prime} \hat{z} G_{A}^{z z}
$$

The key steps in development of LF approximation of Green's functions for formulation A are provided in Appendix B. Components of magnetic vector potential, for source and evaluation points in same layer with index $m$, are expressed as:

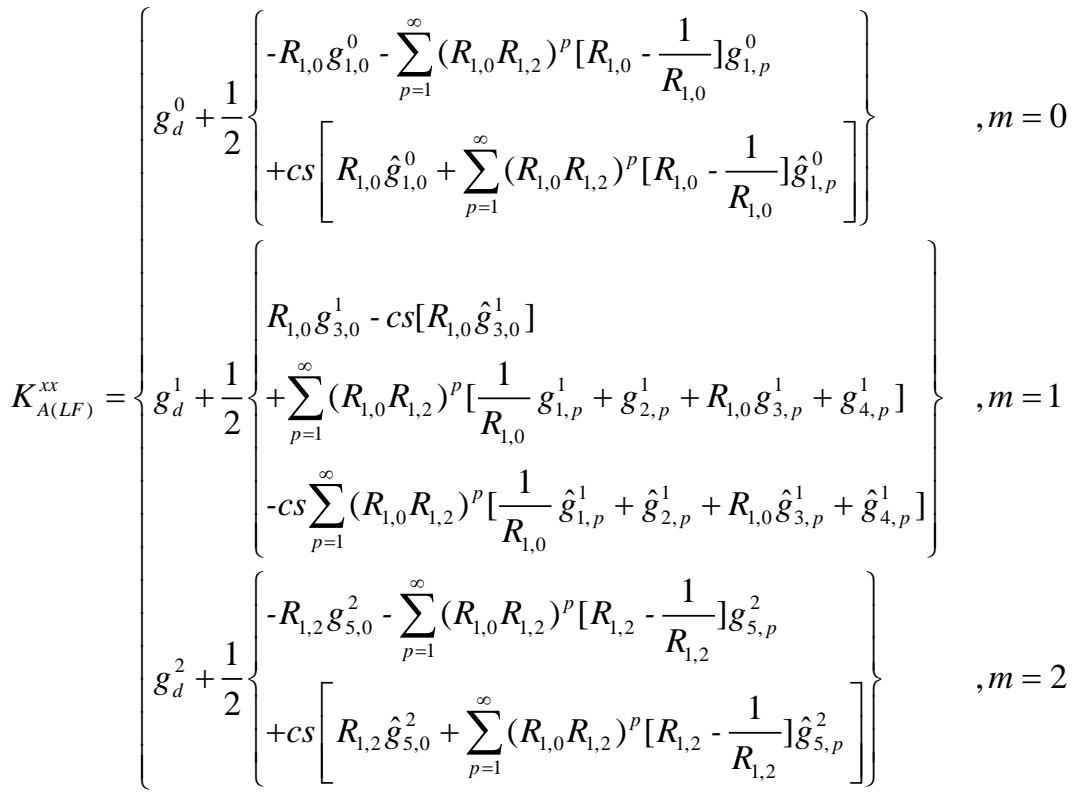

$$
\begin{aligned}
& K_{A(L F)}^{y x}=\left\{\begin{array}{lll}
\frac{1}{2} s n\left[R_{1,0} \hat{g}_{1,0}^{0}+\sum_{p=1}^{\infty}\left(R_{1,0} R_{1,2}\right)^{p}\left[R_{1,0}-\frac{1}{R_{1,0}}\right] \hat{g}_{1, p}^{0}\right] & , m=0 \\
-\frac{1}{2} s n\left[\sum_{1,0} \hat{g}_{3,0}^{1}+\right. & , m=1 \\
\frac{1}{2} s n\left[R_{1,0} R_{1,2}\right)^{p}\left[\frac{1}{R_{1,0}} \hat{g}_{1, p}^{1}+\hat{g}_{2, p}^{1}+R_{1,0} \hat{g}_{3, p}^{1}+\hat{g}_{4, p}^{1}\right] & , m=2
\end{array}\right.
\end{aligned}
$$

Note that $K_{A(L F)}^{x z}=K_{A(L F)}^{y z}=0, K_{A(L F)}^{y y}$ is similar to $K_{A(L F)}^{x x}$, but with changed sign before the $c s$ term, while $G_{A(L F)}^{z z}$ and $G_{\Phi(L F)}$ are identical with (8) and (9), respectively. In above equations, terms $c s$ and $s n$ are for $\cos (2 \varphi)$ and $\sin (2 \varphi)$. 


\section{Comparison of Accuracy of the LF Approximations}

Accuracy of the proposed and existing LF approximations is compared for a set of numerical tests. We consider four cases, illustrated on Figs. 2-5:

- Energized 10-m long horizontal wire

- Passive 5-m long horizontal wire that parallels the energized wire

- Passive 5-m long horizontal wire that is perpendicular to the energized wire

- Passive 1.5-m long vertical wire near the horizontal energized wire

All wires in Figs. 2-5 are with $7 \mathrm{~mm}$ radius and are buried at a depth of $0.5 \mathrm{~m}$ in two-layer earth, with thickness $d=2.5 \mathrm{~m}$ of the earth's upper layer. Characteristics of the two-layered earth are expressed in terms of the reflection factor $K$ [16]:

$$
K=\left(\rho_{2}-\rho_{1}\right) /\left(\rho_{2}+\rho_{1}\right)
$$

where the resistivity of the top earth layer is fixed to either $\rho_{1}=100 \Omega \mathrm{m}$ or $\rho_{1}=1000 \Omega \mathrm{m}$, and the resistivity of the bottom layer is varied accordingly to $K=-0.9,0.0$ or +0.9 . Relative permittivities and permeabillities are set to $\varepsilon_{\mathrm{r} 1}=\varepsilon_{\mathrm{r} 2}=10$ and $\mu_{\mathrm{r} 1}=\mu_{\mathrm{r} 2}=1$, respectively. In the case of the passive vertical wire in Fig. 5, its upper point is at a depth of $0.5 \mathrm{~m}$. The wires are energized by a harmonic voltage generator with an RMS value of $1 \mathrm{~V}$ connected serially at the midpoint of the $10 \mathrm{~m}$ horizontal wire.

Figs. 2-5 show the computed error for the longitudinal current distribution obtained by the new LF approximation and the existing image model based on traditional formulation of potentials, with reference to results obtained by commercial electromagnetic simulation software FEKO [12]. RMS error for the longitudinal current along the conductor [17] is computed as follows:

$$
\varepsilon_{\mathrm{RMS}}=\left[\frac{\sum_{n=1}^{N}\left|\hat{I}_{n}^{A}-\hat{I}_{n}^{R}\right|^{2}}{\sum_{n=1}^{N}\left|\hat{I}_{n}^{R}\right|^{2}}\right]^{1 / 2} \cdot 100(\%)
$$

Here, $\hat{I}_{n}^{R}$ is the phasor of the current samples along the conductor computed by rigorous model, and $\hat{I}_{n}^{A}$ is the phasor of the current samples obtained using approximate solutions. $N$ is total number of segments along the conductor. 

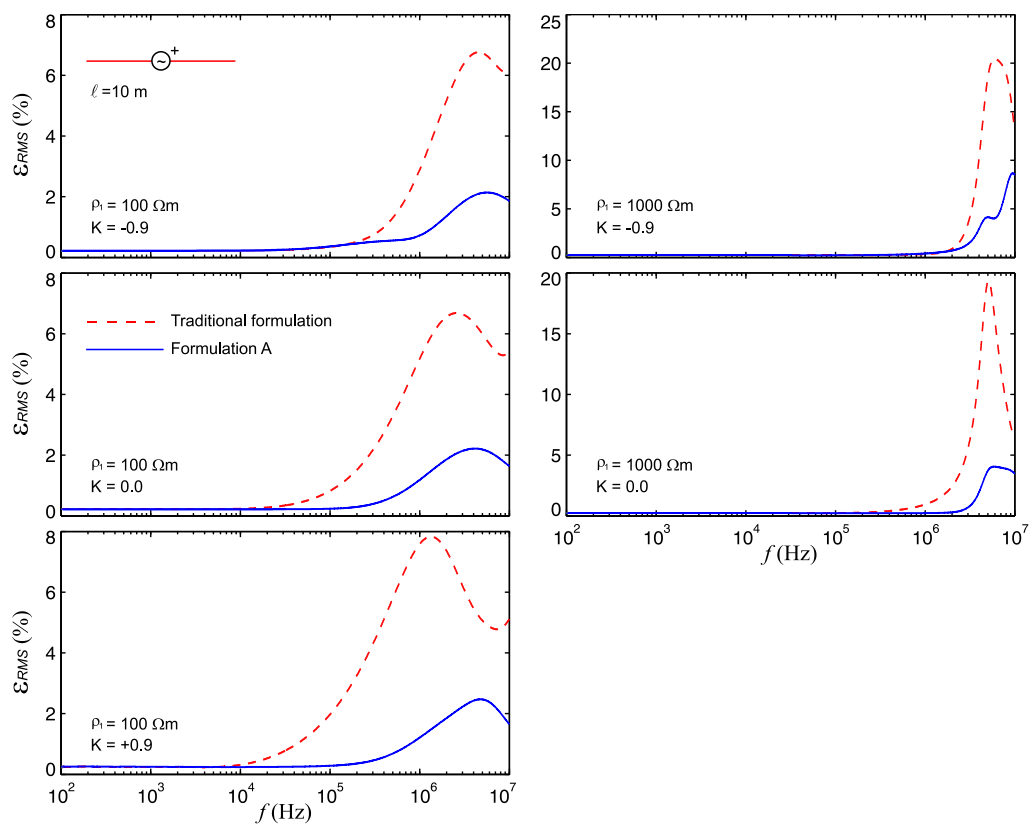

Figure 2

$\varepsilon_{\mathrm{RMS}}$ error for currents in energized 10 -m long horizontal wire
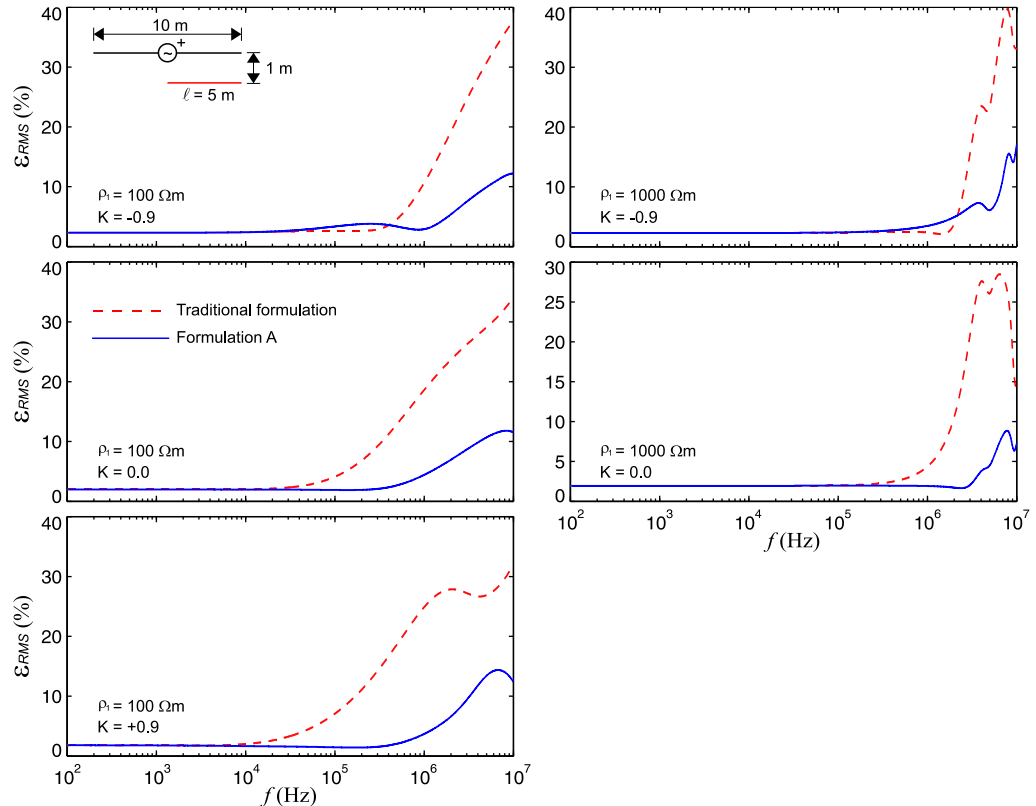

Figure 3

$\varepsilon_{\mathrm{RMS}}$ error for currents in passive 5-m long horizontal wire that parallels energized wire 

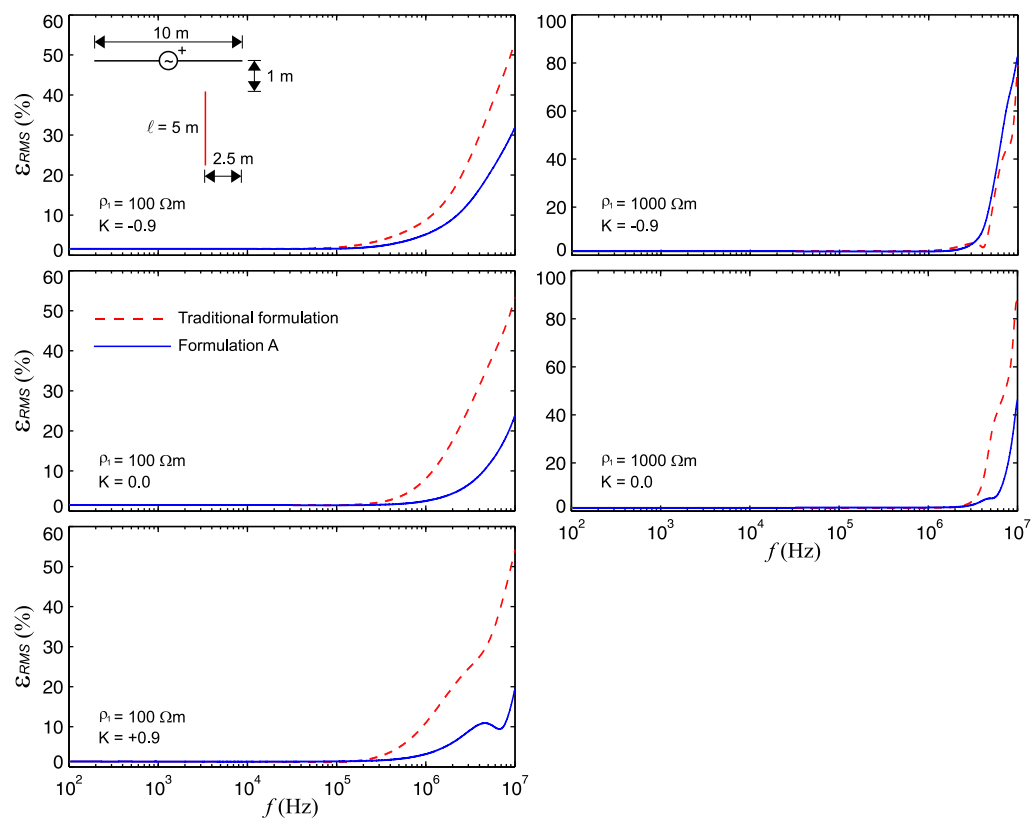

Figure 4

$\varepsilon_{\mathrm{RMS}}$ error for currents in passive 5-m long horizontal wire that is perpendicular to energized wire
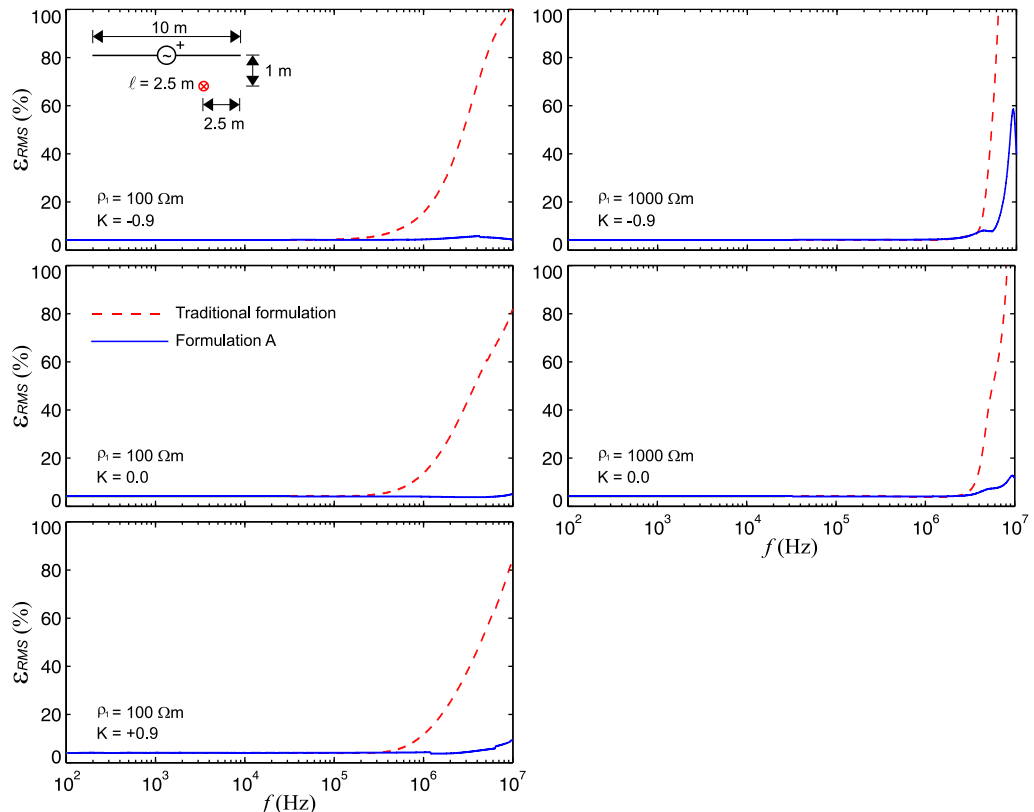

Figure 5

$\varepsilon_{\mathrm{RMS}}$ error for currents in passive 1.5 -m long vertical wire near horizontal energized wire 


\section{Conclusions}

This paper provides LF approximations for the electromagnetic modeling of wires above or within a two-layered earth, based on the alternative choice of potentials. Accuracy of the proposed approximation is validated for source and observation points within the earth's upper layer and for a range of different wire geometries, frequencies and soil characteristics.

The results illustrated in Figs. 2-5, show that both approximations provide good and nearly equal accuracy for frequencies up to $10 \mathrm{kHz}$. However, the proposed LF approximation derived from formulation A, is more accurate than existing LF approximation derived from the traditional formulation, for frequencies within the range $10 \mathrm{kHz}-10 \mathrm{MHz}$, for nearly all cases. Such improvement of accuracy may widen the application of LF approximations in transient analysis where currents with significant high frequency contents are involved, such as, those related to subsequent lightning strikes or due to manipulations in the electrical power systems. The introduced error in transient analysis is not within the scope of this paper, but its evaluation will be considered as a continuation of this work in the future.

The paper also provides a mathematically exact solution for the spatial domain Green's functions for formulation A, for source and observation points within the same layer of general planar-layered media, cast in a form which is appropriate for the development of the proposed LF approximation.

\section{Appendix A - Exact Green's Functions for Formulation A in Planar-Layered Media}

Exact form of Green's functions for formulation A of potentials in MPIE, for source and observation points within same layer, in terms of transmission line parameters and in spectral domain, can be found in [11]. Here, Green's functions are cast in a form that is more appropriate for development of the proposed approximation:

$$
\begin{aligned}
& K_{A}^{x x}=g_{d}^{m}+\frac{1}{2}\left[I_{1}+\cos (2 \varphi) I_{2}\right] \\
& K_{A}^{y y}=g_{d}^{m}+\frac{1}{2}\left[I_{1}-\cos (2 \varphi) I_{2}\right] \\
& K_{A}^{x y}=K_{A}^{y x}=\sin (2 \varphi) \frac{1}{2} I_{2} \\
& G_{A}^{z z}=g_{d}^{m}+I_{3} \\
& G_{\Phi}=g_{d}^{m}+I_{4}
\end{aligned}
$$

where $g_{d}^{m}$ is direct term related to a spherical wave due to electric dipole in an unbounded medium with characteristics of the layer $m$ : 
$g_{d}^{m}=\frac{e^{j k_{m} r_{d}}}{r_{d}}, \quad r_{d}=\sqrt{\rho^{2}+\left|z-z^{\prime}\right|^{2}}$

and $I_{1}, I_{2}, I_{3}$ and $I_{4}$ are Sommerfeld-type integrals related to the up- and downgoing waves reflected from interfaces, for example at $z_{0}$ and $z_{1}$ illustrated on Fig 1.

$$
\begin{aligned}
& I_{1}=\int_{0}^{\infty}\left[\left(A_{h}-B_{h}\right) \frac{e^{j k_{m, z}\left(z-z^{\prime}\right)}}{j k_{m, z}}+\left(C_{h}+D_{h}\right) \frac{e^{-j k_{m, z}\left(z-z^{\prime}\right)}}{j k_{m, z}}\right] J_{0}\left(k_{\rho} \rho\right) k_{\rho} d k_{\rho} \\
& I_{2}=\int_{0}^{\infty}\left[\left(A_{h}+B_{h}\right) \frac{e^{j k_{m, z}\left(z-z^{\prime}\right)}}{j k_{m, z}}+\left(C_{h}-D_{h}\right) \frac{e^{-j k_{m, z}\left(z-z^{\prime}\right)}}{j k_{m, z}}\right] J_{2}\left(k_{\rho} \rho\right) k_{\rho} d k_{\rho} \\
& I_{3}=\int_{0}^{\infty}\left[A_{v} \frac{e^{-j k_{m, z}\left(z-z^{\prime}\right)}}{j k_{m, z}}+B_{v} \frac{e^{j k_{m, z}\left(z-z^{\prime}\right)}}{j k_{m, z}}\right] J_{0}\left(k_{\rho} \rho\right) k_{\rho} d k_{\rho} \\
& I_{4}=\int_{0}^{\infty}\left[C_{v} \frac{e^{-j k_{m, z}\left(z-z^{\prime}\right)}}{j k_{m, z}}+D_{v} \frac{e^{j k_{m, z}\left(z-z^{\prime}\right)}}{j k_{m, z}}\right] J_{0}\left(k_{\rho} \rho\right) k_{\rho} d k_{\rho}
\end{aligned}
$$

Coefficients $A_{h, v}, B_{h, v}, C_{h, v}$ and $D_{h, v}$, and related parameters are expressed as:

$$
\begin{aligned}
& \tilde{M}_{m}^{T E, T M}=\left[1-\tilde{R}_{m, m-1}^{T E, T M} \tilde{R}_{m, m+1}^{T E, T M} e^{-j k_{m, z} 2 \Delta_{m}}\right]^{-1} \\
& \tilde{R}_{m, m \pm 1}^{T E, T M}=\left(R_{m, m \pm 1}^{T E, T M}+\tilde{R}_{m \pm 1, m \pm 2}^{T E, T M} e^{-j k_{m \pm, z} 2 \Delta_{m \pm 1}}\right) \tilde{M}_{m \pm 1}^{T E, T M} \\
& A_{h}=\tilde{R}_{m, m-1}^{T E} e^{-j k_{m, z}\left(\Delta_{m}-\left(z^{\prime}-z_{m}\right)\right)}\left[e^{-j k_{m, z}\left(\Delta_{m}-\left(z^{\prime}-z_{m}\right)\right)}+\tilde{R}_{m, m+1}^{T E} e^{-j k_{m, z}\left(\Delta_{m}+\left(z^{\prime}-z_{m}\right)\right)}\right] \tilde{M}_{m}^{T E} \\
& B_{h}=-D_{v}=\tilde{R}_{m, m-1}^{T M} e^{-j k_{m, z}\left(\Delta_{m}-\left(z^{\prime}-z_{m}\right)\right)}\left[e^{-j k_{m, z}\left(\Delta_{m}-\left(z^{\prime}-z_{m}\right)\right)}-\tilde{R}_{m, m+1}^{T M} e^{-j k_{m, z}\left(\Delta_{m}+\left(z^{\prime}-z_{m}\right)\right)}\right] \tilde{M}_{m}^{T M} \\
& C_{h}=\tilde{R}_{m, m+1}^{T E} e^{-j k_{m, z}\left(z^{\prime}-z_{m}\right)}\left[e^{-j k_{m, z}\left(z^{\prime}-z_{m}\right)}+\tilde{R}_{m, m-1}^{T E} e^{-j k_{m, z}\left(2 \Delta_{m}-\left(z^{\prime}-z_{m}\right)\right)}\right] \tilde{M}_{m}^{T E} \\
& D_{h}=C_{v}=\tilde{R}_{m, m+1}^{T M} e^{-j k_{m, z}\left(z^{\prime}-z_{m}\right)}\left[-e^{-j k_{m, z}\left(z^{\prime}-z_{m}\right)}+\tilde{R}_{m, m-1}^{T M} e^{-j k_{m, z}\left(2 \Delta_{m}-\left(z^{\prime}-z_{m}\right)\right)}\right] \tilde{M}_{m}^{T M} \\
& A_{v}=\tilde{R}_{m, m+1}^{T M} e^{-j k_{m, z}\left(z^{\prime}-z_{m}\right)}\left[e^{-j k_{m, z}\left(z^{\prime}-z_{m}\right)}+\tilde{R}_{m, m-1}^{T M} e^{-j k_{m, z}\left(2 \Delta_{m}-\left(z^{\prime}-z_{m}\right)\right)}\right] \tilde{M}_{m}^{T M} \\
& B_{v}=\tilde{R}_{m, m-1}^{T M} e^{-j k_{m, z}\left(\Delta_{m}-\left(z^{\prime}-z_{m}\right)\right)}\left[e^{-j k_{m, z}\left(\Delta_{m}-\left(z^{\prime}-z_{m}\right)\right)}+\tilde{R}_{m, m+1}^{T M} e^{-j k_{m, z}\left(\Delta_{m}+\left(z^{\prime}-z_{m}\right)\right)}\right] \tilde{M}_{m}^{T M}
\end{aligned}
$$

In above equations, geometric quantities $z$ and $z$ ' are positions of observation and source points with respect to $z$ - axis with origin at the earth's surface, $\Delta_{\mathrm{m}}$ is the thickness of $m$-th layer and $z_{\mathrm{m}}$ is depth of the interface of the $m$-th and $m+1$-th layers. 
Generalized reflection coefficients $\tilde{R}_{m, m \pm 1}^{T E, T M}$ for layer $m$ are obtained by iterative procedure, starting from the lowermost half-space $n$ (semi infinite earth) for evaluation of $\tilde{R}_{m, m+1}^{T E, T M}$ and the topmost half-space with index 0 (air) for evaluation of $\tilde{R}_{m, m-1}^{T E, T M}$, considering that $\tilde{R}_{n, n+1}^{T E, T M}=0$ and $\tilde{R}_{0,-1}^{T E, T M}=0$, respectively.

Other required parameters are expressed as:

$$
\begin{aligned}
& R_{m, m \pm 1}^{T E}=\frac{\mu_{m \pm 1} k_{m, z}-\mu_{m} k_{m \pm 1, z}}{\mu_{m \pm 1} k_{m, z}+\mu_{m} k_{m \pm 1, z}}, R_{m, m \pm 1}^{T M}=\frac{\underline{\sigma}_{m \pm 1} k_{m, z}-\underline{\sigma}_{m} k_{m \pm 1, z}}{\underline{\sigma}_{m \pm 1} k_{m, z}+\underline{\sigma}_{m} k_{m \pm 1, z}} \\
& \underline{\sigma}_{m}=\sigma_{m}+j \omega \varepsilon_{m}, k_{m}=\sqrt{-j \omega \mu_{m} \underline{\sigma}_{m}}, k_{m, z}=\sqrt{k_{m}^{2}-k_{\rho}^{2}}
\end{aligned}
$$

\section{Appendix B - Development of LF Approximation of Green's Functions for Formulation A}

The LF approximations of Green's functions for formulation A of potentials in MPIE, for a two-layered earth are developed following the procedures provided in [14] [15]. Here, some key steps in development are briefly provided for completeness.

For two-layered earth, generalized reflection coefficients are reduced to:

$$
\begin{aligned}
& M_{1}^{T E, T M}=\left[1-R_{1,0}^{T E, T M} R_{1,2}^{T E, T M} e^{-2 j k_{1,2} d}\right]^{-1} \\
& \tilde{R}_{0,-1}^{T E, T M}=0 ; \quad \tilde{R}_{1,0}^{T E, T M}=R_{1,0}^{T E, T M} ; \quad \tilde{R}_{2,1}^{T E, T M}=\left(R_{2,1}^{T E, T M}+R_{1,0}^{T E, T M} e^{-2 j k_{1,2} d}\right) M_{1}^{T E, T M} ; \\
& \tilde{R}_{2,3}^{T E, T M}=0 ; \quad \tilde{R}_{1,2}^{T E, T M}=R_{1,2}^{T E, T M} ; \quad \tilde{R}_{0,1}^{T E, T M}=\left(R_{0,1}^{T E, T M}+R_{1,2}^{T E, T M} e^{-2 j k_{1, z} d}\right) M_{1}^{T E, T M}
\end{aligned}
$$

where $d$ is thickness of topmost earth layer. The first key simplification is to obtain LF approximation of the reflection coefficients $R_{m, m \pm 1}^{T E}$ and $R_{m, m \pm 1}^{T M}$. For frequencies approaching $0 \mathrm{~Hz}$ the following approximation is valid: $k_{0, \mathrm{z}} \approx k_{1, \mathrm{z}} \approx k_{2, \mathrm{z}}$ since $k_{n}^{2} \rightarrow 0$ for $n=0,1,2$.

Then reflection coefficients become constants and can be extracted from the Sommerfeld integrals. Considering that in practical cases $\mu_{1}=\mu_{2}=\mu_{0}$, the LF approximations of TE and TM related reflection coefficients can be expressed as:

$R_{m, m \pm 1(L F)}^{T E} \rightarrow 0 ; R_{m, m \pm 1(L F)}^{T M} \rightarrow-R_{m, m \pm 1}$ while $M_{1(L F)}^{T E} \rightarrow 1$

The second key simplification is to expand $M_{1(L F)}^{T M}$ in following series [18]:

$$
M_{1(L F)}^{T M} \rightarrow\left[1-R_{1,0} R_{1,2} e^{-2 j k_{1, z} d}\right]^{-1} \approx \sum_{p=0}^{\infty}\left(R_{1,0} R_{1,2}\right)^{p} e^{-j k_{1,2} 2 d p}
$$


Additionally, in development of LF approximations for layers 0 and 2, wave numbers of different layers can appear in equations. In such case it is impossible to obtain simple closed-form approximation of Green's function. To circumvent this problem, third key simplification is introduced, by which, $k_{0, z}, k_{1, z}$ and $k_{2, z}$ are substituted with unique wave number $k_{m, z}$ related to the observation layer.

In the final step of development, following identities are used to obtain closedform solutions of the Green's functions:

$$
\begin{aligned}
& g_{l, p}^{m}=\int_{0}^{\infty} \frac{e^{-j k_{m, z} h_{l, p}}}{j k_{m, z}} J_{0}\left(k_{\rho} \rho\right) k_{\rho} d k_{\rho}=\frac{e^{-j k_{m} r_{l, p}}}{r_{l, p}} \\
& \hat{g}_{l, p}^{m}=\int_{0}^{\infty} \frac{e^{-j k_{m, z} h_{l, p}}}{j k_{m, z}} J_{2}\left(k_{\rho} \rho\right) k_{\rho} d k_{\rho}=\frac{2\left(e^{-j k_{m} h_{l, p}}-e^{-j k_{m} r_{l, p}}\right)}{j k_{m} \rho^{2}}-\frac{e^{-j k_{m} r_{l, p}}}{r_{l, p}} \\
& r_{l, p}=\sqrt{\rho^{2}+h_{l, p}^{2}}, l=1,2,3,4 ., h_{1, p}=2 d p+\left(z+z^{\prime}\right), h_{2, p}=2 d p+\left(z-z^{\prime}\right), \\
& h_{3, p}=2 d p-\left(z+z^{\prime}\right), h_{4, p}=2 d p-\left(z-z^{\prime}\right) .
\end{aligned}
$$

where subscript $l$ is related to the vertical distance $h_{l, p}$ between the observation point and source image with index $p$, from the infinite series of images.

Note that when $\sigma_{1}=\sigma_{2}, \varepsilon_{\mathrm{r} 1}=\varepsilon_{\mathrm{r} 2}$ and $\mu_{1}=\mu_{2}=\mu_{0}$, image approximations can be further reduced to ones valid for uniform earth, proposed in [10].

\section{References}

[1] J. He, R. Zeng and B. Zhang: Methodology and Technology for Power System Grounding, New York: Wiley, 2013

[2] E. B. Joffe, K-S Lock: Grounds for Grounding: A Circuit to System Handbook, New York: Wiley, 2011

[3] Y. Baba, R. A. Rakov: Electromagnetic Computation Methods for Lightning Surge Protection Studies, New York: Wiley, 2016

[4] R. W. P. King and G. S. Smith: Antennas in Matter, Cambridge, MA: MIT Press, 1981

[5] K. A. Michalski: The mixed-potential electric field integral equation for objects in layered media, Archiv für elektronik und übertragungstechnik, Vol. 39, No. 5, Sep./Oct. 1985, pp. 317-322

[6] R. F. Harrington: Field Computation by Moment Methods, New York: Macmillan, 1968; New York: Wiley, 1993

[7] A. Sommerfeld: Partial Differential Equations in Physics, Chapter VI, New York: Academic Press, 1949 
[8] A. Erteza and B. K. Park: Non-uniqueness of resolution of Hertz vector in presence of a boundary, and a horizontal dipole problem, IEEE Trans. Antennas Propagat., Vol. AP-17, May 1969, pp. 376-378

[9] K. A. Michalski: On the scalar potential of a point charge associated with a time-harmonic dipole in a layered medium, IEEE Trans. Antennas Propagat., Vol. AP-35, Nov. 1987, pp. 1299-1301

[10] B. Markovski, L. Grcev, V. Arnautovski-Toseva: Accurate low-frequency approximation for wires within a conducting half-space, IEEE Trans. Electromagn. Compat., doi: 10.1109/TEMC.2018.2881932, pp. 1-4

[11] K. A. Michalski and D. Zheng: Electromagnetic scattering and radiation by surfaces of arbitrary shape in layered media, Part I: Theory, IEEE Trans. Antennas Propagat., Vol. 38, No. 3, Mar. 1990, pp. 335-344

[12] EM Software and Systems-S.A. (Pty) Ltd., FEKO, Stellenbosch, South Africa, 2009 [Online] Available: http://www.feko.info

[13] G. Dural, M. I. Aksun: Closed-form Green's functions for general sources and stratified media, IEEE Trans. on Microwave Theory and Techniques, Vol. 43, No. 7, Jul. 1995, pp. 1545-1552

[14] V. Arnautovski-Toseva and L. Grcev: Electromagnetic analysis of horizontal wire in two-layered soil, J. Comput. Appl. Math., Vol. 168, Nos. 1-2, Jul. 2004, pp. 21-29

[15] V. Arnautovski-Toseva, L. Grcev: Image and exact models of a vertical wire penetrating a two-layered earth, IEEE Trans. Electromagn. Compat, Vol. 53, No. 4, Nov. 2011, pp. 968-976

[16] F. Dawalibi, D. Mukhedikar: Influence of ground rods on grounding grids, IEEE Trans. on Power Apparatus and Systems, Vol. PAS-98, No. 6, Nov./Dec. 1979, pp. 2089-2097

[17] A. Poggio, R. Bevensee and E. K. Miller: Evaluation of some thin wire computer programs, IEEE Antennas Propag. Symp., Vol. 12, Jun. 1974, pp. $181-184$

[18] L. M. Brekhovskikh, Waves in Layered Media. New York: Academic, 1960 